\title{
Study design flaws and statistical challenges in evaluating fertility treatments
}

\author{
Jack Wilkinson and Katie Stocking \\ ${ }^{1}$ Centre for Biostatistics, Division of Population Health, Health Services Research and Primary Care, Faculty of Biology, Medicine, and Health, University of \\ Manchester, Manchester, UK
}

Correspondence should be addressed to J Wilkinson: jack.wilkinson@manchester.ac.uk

\begin{abstract}
Health interventions should be tested before being introduced into clinical practice, to find out whether they work and whether they are harmful. However, research studies will only provide reliable answers to these questions if they are appropriately designed and analysed. But these are not trivial tasks. We review some methodological challenges that arise when evaluating fertility interventions and explain the implications for a non-statistical audience. These include flexibility in outcomes and analyses; use of surrogate outcomes instead of live birth; use of inappropriate denominators; evaluating cumulative outcomes and time to live birth; allowing each patient or couple to contribute to a research study more than once. We highlight recurring errors and present solutions. We conclude by highlighting the importance of collaboration between clinical and methodological experts, as well as people with experience of subfertility, for realising high-quality research.
\end{abstract}

\section{Lay summary}

We do research to find out whether fertility treatments are beneficial and to make sure they don't cause harm. However, research will only provide reliable answers if it is done properly. It is not unusual for researchers to make mistakes when they are designing research studies and analysing the data that we get from them. In this review, we describe some of the mistakes people make when they do research about fertility treatments and explain how to avoid them. These include challenges which arise due to the large number of things that can be measured and reported when looking to see if fertility treatments work; failure to check whether the treatment increases the number of live births; failing to include all study participants in calculations; challenges in studies where participants may have more than one treatment attempt. We conclude by highlighting the importance of collaboration between clinical and methodological experts, as well as people with experience of fertility problems.

Key Words: - assisted reproduction - reproductive medicine

Reproduction and Fertility (2021) 2 C9-C21

\section{Introduction}

It is hopefully not too controversial to suggest that fertility interventions should be robustly tested before they are introduced into routine practice. In the absence of robust testing, it cannot be known whether treatment improves fertility outcomes, or even worsens them (Braakhekke et al. 2017a, Wang et al. 2020). The challenge is to distinguish the subtle effects of an intervention from the plethora of other factors, most of which are unknown, that determine whether or not a patient will successfully conceive and have a child. In principle, randomisation of a sufficient

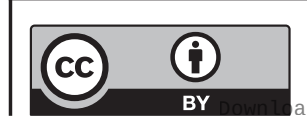


number of participants allows us to achieve this goal (Yusuf et al. 1984, Wilkinson et al. 2019a). It might be possible to discern larger effects (e.g. those which lead to a substantial improvement in live births) without randomisation. However, without randomisation, we usually can't tell whether any differences in outcome are actually due to other things which we have not, perhaps cannot, measure and adjust for. Regardless of whether or not randomisation is used, the reliability of a study's results is dependent on good research design and execution, as well as appropriate data analysis and interpretation. The purpose of this review is to draw attention to some common pitfalls when using quantitative methods to evaluate fertility interventions.

Before we begin, we note that the following discussion is far from comprehensive. We have restricted our focus to issues that arise due to the nature of subfertility and its therapies. There are many other methodological fallacies which commonly occur in biomedical research irrespective of the clinical context. We do not cover these here, but point readers to more general resources in the section titled Conclusions. We have also opted to focus on the evaluation of interventions, and so do not cover methodological issues relating to the development of diagnostic or prognostic tools. We caution the reader that different considerations apply in that context, and again we point to some resources at the end of the article. Finally, we acknowledge that we are not the first to write a review of statistical issues arising in fertility research, with earlier examples focussing on trials (Vail \& Gardener 2003) or observational studies (Messerlian \& Gaskins 2017, Dodge et al. 2020). We have neither selected nor avoided topics based on these previous review articles. Instead, we have selected topics which we believe are consequential and have tried to produce a clear exposition of each with a nonstatistical audience in mind.

\section{Multiple treatment stages introduce multiple methodological challenges}

Many methodological challenges in fertility research arise due to the fact that the treatments under study are sequential in nature, involving multiple stages. For example, assisted reproduction typically involves ovarian hyperstimulation, fertilisation of the oocytes, culture of the resulting embryos, and selection of the best for transfer. Surplus embryos might be frozen and transferred in subsequent cycles. Even natural conception, involving no additional medical care, is typically evaluated over a period of several months, with multiple opportunities to get pregnant. Multistage treatments of this sort present a variety of options to the researcher when deciding both what to measure and who to measure it in. As we will explain, however, many of these options do not represent methodologically sound strategies.

\section{The problem of many outcomes}

The performance of multistage-assisted reproductive treatments can be quantified at each stage. For example, the response to ovarian hyperstimulation, the number and quality of embryos obtained following fertilisation and culture, success of the embryo transfer, the outcome of pregnancy and health of any resulting offspring can all be measured and reported in a study involving in vitro fertilisation (IVF) (Heijnen et al. 2004). Accordingly, a recent review of outcome reporting in infertility RCTs found that many different outcomes (361 numerators and 87 denominators, resulting in 815 distinct combinations) appear in the literature, with a median (interquartile range) of 11 (7 to 16) reported per study (Wilkinson et al. 2016).

Reporting multiple outcomes of treatment in a study are not a problem in and of itself and can serve to give a fuller description of how an intervention influences live birth rates. Additionally, reporting metrics relating to safety, such as ovarian response to hyperstimulation and neonatal outcomes, is essential in order to permit the detection of potential harms. Nonetheless, the availability of an expansive menu of outcomes poses a threat to a study's statistical validity if not handled appropriately. In particular, challenges arise when many statistical tests are performed (multiple testing) (Moher et al. 2010), or when results are selectively reported on the basis of the statistical test result (selective outcome reporting). (Furukawa et al. 2007, Al-Marzouki et al. 2008, Dwan et al. 2014) Unfortunately, the wide array of outcomes arising from fertility treatments means that there are lots of things to test and plenty of reporting options to choose from (Braakhekke et al. 2017b). Moreover, results may be reported for various subgroups of participants, compounding these problems. When multiple tests are performed, the chance of obtaining a statistically significant result under the null (e.g. a $P$-value $<0.05$ when no effect exists) will be greater than 5\% ( Farland et al. 2016, Roberts \& Vail 2017). As a result, researchers may claim to have found evidence of an effect when in reality the play of chance represents a suitable explanation. Selective outcome reporting gives a misleading impression of the effect of a treatment in an individual study and also distorts the results of meta-analyses.

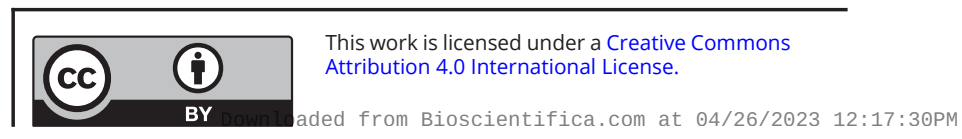


In order to protect against these concerns, it is advisable to have a single outcome measure prespecified as the primary outcome of the study (Moher et al. 2010). A statistical test of this outcome can then be used as the basis for the study conclusion. Statistical tests of secondary outcomes should also be prespecified and ideally limited in number, but even with these measures in place, they should be interpreted with caution. This principle introduces a tension between the desire to give a comprehensive account of the treatment process on the one hand and the desire to safeguard the credibility of statistical comparisons on the other (Althouse 2016). A pragmatic approach might be to fully report the procedural outcomes of treatment while restricting statistical testing to a small number of hypotheses relating to the postulated mechanism of the intervention effect; another approach is to adopt a hierarchical testing strategy (European Medicines Agency 2016). An example of a reasonably compelling finding arising from the analysis of a secondary outcome is the reduction in miscarriage using hyaluronan-based sperm selection for intracytoplasmic sperm injection (ICSI) (Miller et al. 2019). This example was prespecified, the number of tests performed was reasonably modest, and the corresponding test clearly indicated that the data were incompatible with the null hypothesis of no effect on miscarriage, with a $P$-value of 0.003 .

Although strict prespecification of outcomes and analyses is a powerful strategy for maintaining the credibility of statistical inferences, outside of RCTs, this is not common practice in fertility research. The reasons for this might include a lack of awareness of the frailty of results obtained via data-driven statistical analyses (Simmons et al. 2011) and a lack of awareness of mechanisms and platforms for formal prespecification. Another reason might relate to the fact that a study is more likely to be published if it has a statistically significant finding (resulting in so-called publication bias (Easterbrook 1987, Easterbrook et al. 1991)). Since it is much easier to achieve statistical significance (albeit, of a spurious nature) via data-dredging than by performing a valid test of a prespecified hypothesis, the latter option is likely to appear less attractive. An innovative publication type, known as a Registered Report, represents a solution to this problem ( Chambers 2019, Chambers et al. 2015). In a Registered Report, authors submit a protocol for their research project to a journal for peer review, including the specific hypotheses to be tested, before the study has been initiated. The journal will make a decision regarding whether or not to publish the findings of the research at this stage. This means that publication is determined by the importance of the research question and the robustness of the methods rather than on the basis of the study results. The guarantee of publication removes the incentive for researchers to find (really, create the illusion of) statistically significant results, and the prospective declaration of hypotheses permits statistically sound testing to be conducted. This format has been adopted by some fertility journals (Wilkinson et al. 2019b) but is yet to gain a foothold in the field, despite the potential benefits for the evidence base.

\section{Diversity of definitions}

The problems associated with variation in reporting in fertility research are exacerbated by the fact that multiple definitions are in use for some outcomes (Wilkinson et al. 2016). To illustrate, a review identified numerous definitions of biochemical pregnancy (23), clinical pregnancy (61), ongoing pregnancy (20) and live birth (7) (Wilkinson et al. 2016). This expands the array of reporting options. Concerningly, selective reporting of different versions of one outcome might be more difficult to detect than selection or omission of an outcome altogether. For example, suppose a research team finds an effect of a treatment on live birth if they include only birth events of at least 28 weeks gestation, but not if they include all events after 24 weeks gestation, and therefore, decides to use the former definition. This would be much less obvious than a study omitting live birth altogether.

An additional consequence of heterogeneity in outcome reporting is that it is difficult to compare and combine results of studies which report different outcomes or use different definitions of the same outcomes. The inability to include good-quality data in meta-analysis is a serious hindrance to progress in fertility research. This was highlighted by a recent review which found that metaanalyses of RCTs of fertility interventions were frequently uninformative, in part due to a lack of available data (Stocking et al. 2019). The recent publication of a core outcome set for infertility is intended to tackle outcome heterogeneity by specifying a minimum set of outcomes that should be routinely reported, using standardised definitions (Duffy et al. 2020). The success of this initiative is likely to depend on the commitment of journals to monitoring and enforcing adherence.

\section{Measuring success and surrogate outcomes}

We have described the plurality of measures available for the purpose of evaluating interventions. How can the fertility researcher choose amongst them? The matter of

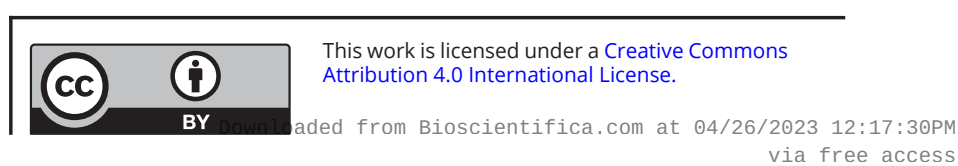


measuring the performance of fertility treatments has been frequently debated over the past two decades (Heijnen et al. 2004, Min et al. 2004, Abdalla et al. 2010, Wilkinson et al. 2017, Goodman et al. 2020). Since people undertake fertility treatments with the goal of having a child, it is generally agreed that live birth is the most appropriate measure for the purpose of evaluating whether an intervention is clinically effective (Harbin Consensus Conference Workshop Group 2014). As such, live birth (Duffy et al. 2020) should generally be used as the primary outcome in studies aiming to evaluate clinical effectiveness (although Braakhekke and colleagues (Braakhekke et al. 2014) have made a reasonable argument for viable pregnancy of 12 weeks duration, on the grounds that pregnancy losses after this time are low).

However, we would emphasise that primary outcomes other than live birth are likely to be more appropriate when the objective is not to evaluate clinical effectiveness. For example, where an experimental intervention has a clear, postulated mechanism of effect, it can be more efficient and more ethical to design a study to test that mechanism before moving to a definitive clinical evaluation. To illustrate, consider a hypothetical novel ovarian stimulation protocol intended to increase the number of oocytes retrieved in predicted poor responders in IVF. Far fewer participants would be required to conduct a well-powered study if number of oocytes, rather than live birth, were used as the primary outcome. If the study ruled out an effect on oocyte yield, there would be no need to proceed to a larger study evaluating live birth, preventing the needless recruitment and allocation of many women to receive an ineffective treatment. A related idea that has been proposed in other clinical fields and which warrants investigation in fertility is to use upstream (or surrogate) outcomes as early indicators of futility during a trial. This involves using the data accrued up to that point to predict whether the trial is likely to identify a benefit of treatment. If not, we can stop the trial. Using a surrogate outcome, rather than waiting for live birth data to become available, would speed up this process (Bratton et al. 2013, Friede et al. 2020).

Nonetheless, it is important to note that the converse does not hold; while failure to improve a well-selected surrogate outcome might be sufficient for the purposes of deserting an intervention, improvement in a surrogate cannot necessarily be taken as proof that the overall clinical outcome (in fertility, usually live birth) would also be improved. Illustrating the point, Svensson and colleagues compiled a list of drugs which had been approved on the basis of a surrogate endpoint but were later shown to be deleterious (Svensson et al. 2013). Nonetheless, endorsing a treatment on the basis of a surrogate outcome appears to be a common fallacy in fertility research. For example, reductions in total fertilisation failure (TFF) are used as justification for using ICSI for non-male factor infertility. However, it is not clear that any advantage in terms of fertilisation translates to improvements in a live birth (Van Rumste et al. 2003). Caution would be required even if the evidence for ICSI reducing TFF in non-male factor infertility was robust. In reality, some of the trials indicating an improvement in TFF are within-person designs, where each participant's oocytes have been randomly divided into ICSI and IVF groups. These designs will exaggerate improvements in TFF compared to clinical practice because the oocyte pools under consideration are half the size.

Surrogate outcomes can be seductive, particularly when there is evidence of correlation between the surrogate and the overall outcome. For example, studies investigating the 'optimal' number of oocytes following ovarian stimulation on the basis of correlation with live birth (Law et al. 2019, 2021, Sunkara et al. 2011) are sometimes misinterpreted as showing that achieving this optimum will maximise the chance of treatment success. But this doesn't follow because a correlation between oocytes and live birth does not actually tell us how changing the number of oocytes changes the chance of having a baby. Accordingly, the observed correlation cannot be interpreted as evidence in favour of a treatment strategy which involves aiming for this optimal oocyte yield. In fact, the data would be consistent with (which is not to say supportive of) a disadvantage of this strategy; perhaps, the strategy would adversely impact the receptivity of the uterine environment in fresh transfers, reducing live births (for example). The effectiveness (and safety) of the strategy would require evaluation in a suitable comparative study.

The relationships between surrogate outcomes and live birth in fertility research appear to require further study. Some surrogate outcomes may indeed be valid, meaning that treatment effects on the surrogate reliably predict treatment effects on live birth. Established methodology exists to investigate the validity of surrogates (Bujkiewicz et al. 2016, Ciani et al. 2017). At present, effects on surrogate outcomes are used to advertise add-on treatments on IVF clinic websites (Van de Wiel et al. 2020, Lensen et al. 2021). Both patients and providers might be unclear about what this does or doesn't mean for the add-on's effect on live birth, and in general, we would caution against counting chickens before they hatch. 


\section{Choosing denominators}

A further complication introduced by multistage treatments relates to the possibility of using different denominators when calculating outcome measures. For example, in ART, live birth can be calculated with a variety of denominators, including per cycle started, per oocyte collection, and per embryo transfer. Debates around the choice of denominator often revolve around relevance to patients and clinicians; for example, it might be argued that live birth per cycle started is usually more relevant than live birth per transfer procedure, because the former incorporates any effect the intervention has on the likelihood that a transfer will take place. To illustrate, preimplantation genetic testing for aneuploidy (PGT-A) might appear superior to morphological embryo selection when success rates are calculated per transfer procedure but not when they are calculated per cycle started (or for that matter, per oocyte retrieval), because PGT-A reduces the chance that a transfer procedure will take place (Homer 2019, Munne et al. 2019). Proponents of PGT-A might respond that the per embryo transfer denominator is nonetheless relevant because it answers the question of whether treatment improves outcomes in women who undergo a transfer procedure.

While the relevance of the research question to stakeholders is a critical consideration, an equally important concern is whether the methods employed actually answer that question. And in general, using an event which occurs after the experimental intervention has been administered to create the denominator (in this example, embryo transfer occurs after PGT-A) will not provide a valid assessment of the intervention effect. The problem is easiest to understand in the context of RCTs but applies to observational studies also. In an RCT comparing two interventions, randomisation confers comparability of the groups; while differences in patient characteristics are inevitable, the fact that they have arisen by chance allows for valid inferences to be drawn. When we use an event which occurs after randomisation as the denominator, we restrict the analysis to a post-randomisation (or 'improper') subgroup of participants (Yusuf et al. 1991, Hirji \& Fagerland 2009). An example would be the subgroup of participants who undergo an embryo transfer procedure in a trial where randomisation took place earlier in the treatment process. However, while randomisation confers comparability of treatment arms in the full randomised cohort, this is not true for the post-randomisation subgroup. On the contrary, whenever treatment influences inclusion in the subgroup (for example, by affecting the likelihood of undergoing an embryo transfer) we expect patient characteristics to systematically differ between treatment arms, essentially a selection bias. Differences in outcome between arms in the subgroup will then reflect any differences in prognosis due to these characteristics and do not represent the effect of treatment. Accordingly, as illustrated in Fig. 1, an intervention could appear more effective 'per embryo transfer' simply by making it more difficult to proceed to a transfer procedure, thereby eliminating patients of worse prognosis from consideration. This would be an unusual interpretation of the notion of therapeutic benefit, presenting a challenge to the idea that this analysis is more relevant to patients.

Proponents of PGT-A might respond that Fig. 1 is not a fair representation of what happens when this intervention is used. In Fig. 1, the implication is that some of the people who didn't undergo embryo transfer would have had a baby had they done so. By contrast, proponents may say that the advantage of PGT-A is not that it improves live birth rates, but rather that it reliably predicts failed embryo transfers, such that it prevents futile transfers and spares patients the experience of miscarriage. This framing implies that the prediction of transfer failure is near perfect, that PGT-A could not influence an embryo's chances of implanting or

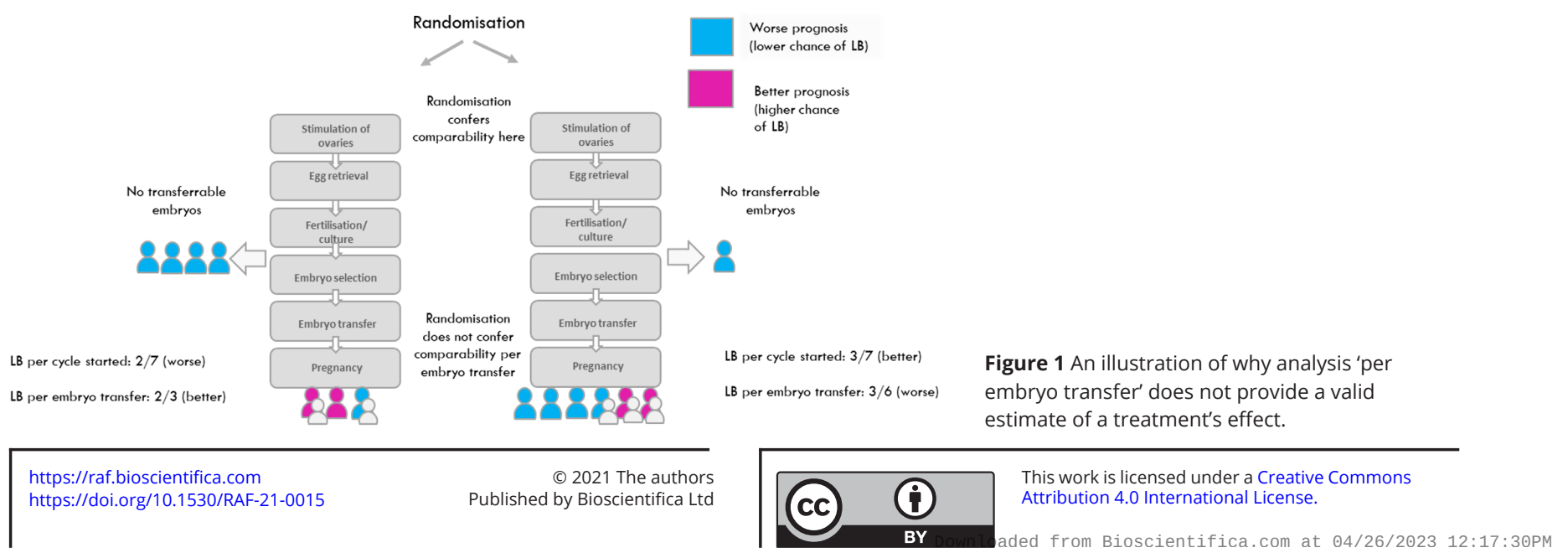

https://raf.bioscientifica.com

https://doi.org/10.1530/RAF-21-0015 (c) 2021 The authors

ded from Bioscientifica.com at 04/26/2023 12:17:30PM 
being carried to term, and that there exist patients who would be willing to pay to reduce the chance of miscarriage without improving (and potentially decreasing) their chance of live birth. It is also curious that if the primary motivation for PGT-A is not to improve live birth but to reduce miscarriage, that RCTs of PGT-A have been designed to demonstrate superiority with respect to live birth rates. A more appropriate design to demonstrate the claim would evaluate noninferiority of PGT-A with respect to live birth while demonstrating a reduction in miscarriage per woman randomised. A noninferiority study aims to show that the intervention is not materially worse than the control (often the standard treatment).

These considerations do not imply that 'per cycle started' should always be used as the denominator. In many situations, an alternative denominator might be preferred, provided that a post-intervention denominator is avoided. As a general rule, there are advantages of beginning follow-up (in an RCT, performing randomisation) of participants as close to the delivery of the intervention under study as possible. For example, RCTs of intrauterine administration of hCG around the time of embryo transfer have randomised participants before the start of treatment, on the day of oocyte collection, and on the day of embryo transfer (Craciunas et al. 2018). Randomising on the day of embryo transfer reduces the scope for randomised participants to drop out from the study before receiving the experimental intervention, allowing for a clearer interpretation of any observed differences in outcome and reducing the required sample size. The randomised woman should then be used to form the denominator for live birth.

\section{Analysing infant outcomes (sometimes there are no good denominators)}

An important question when evaluating fertility interventions is whether and how they affect the health of offspring. This introduces some difficult statistical questions because by definition infant outcomes are only defined for the subset of participants who have a baby, and as we noted in the previous section, restricting comparisons to a post-intervention subgroup is best avoided. For example, an RCT considered the impact of using different embryo culture media in ART on the birthweights of infants (Kleijkers et al. 2016). The appropriate course of action here is the subject of debate, with some methodologists arguing that simple comparisons of outcomes in the subgroup of people having babies are most appropriate (Snowden et al. 2020) and others insisting that bespoke causal inference methods are required (Chiu et al. 2020). The former camp has criticised the assumptions required to use the methods endorsed by the latter, although we note that the simple approach also entails assumptions; for example, it appears to require that the participants are representative of the target population, which is not usually a requirement for valid assessment of a treatment (Bradburn et al. 2020). We acknowledge the controversy but cannot resolve the matter here. Software has recently been made available to assist researchers in thinking about this issue when designing and interpreting fertility trials where outcomes are only defined in a subset of participants (Wilkinson et al. 2020), and interested readers are advised to peruse the reference list of that article.

\section{Evaluating treatment outcomes over multiple treatment attempts}

Because a minority of people undergoing treatment have babies on their first try, a course of fertility treatment often involves multiple attempts to conceive. Many fertility interventions are expected to influence not only the outcome of the next attempt but also any subsequent attempts. For example, in a trial of endometrial scratching for unexplained infertility, the intervention was delivered in the first 12 days of the menstrual cycle, and the effect on natural conception rates over three cycles was evaluated (Lensen et al. 2016). Indeed, some interventions will affect the number of attempts that can be made. For example, any intervention which increases the number of embryos available for freezing following one ovarian stimulation cycle in ART will increase the potential number of embryo transfers in one full cycle. And in some cases, the intervention under study is itself a strategy comprising multiple attempts. For example, a trial of intrauterine insemination (IUI) compared to IVF is being conducted which will compare outcomes following up to four cycles of IUI with those following one full cycle of IVF (Prentice et al. 2020).

As these examples illustrate, in many cases it will be important to evaluate patient outcomes following a course of treatment, rather than restricting focus to the initial attempt(Heijnen et al. 2004). Broadly speaking, there are two possible approaches to doing this. The first is to consider the effect of an intervention on cumulative live birth rates (CLBR), a term which refers to the proportion of patients having a live birth following some maximum number of treatment attempts or after a given period of follow-up time (Malizia et al. 2009). The second is to consider the effect on time to live birth, which refers to how 
long it takes to have a live birth (Sunkara et al. 2020). The two concepts are quite closely related, and in fact, CLBR may be (and often is) estimated using methods for time-toevent analysis.

One analytic challenge which is common to both approaches is the matter of handling participants for whom the outcome status at the end of the follow-up period is unknown. In a routinely collected health dataset, this might happen because the patient ceased treatment before having a baby (either due to their own wishes or at the recommendation of a clinician). In the context of a prospective study, outcome data will be missing whenever a participant is lost to follow-up before having a baby. Assumptions must be made about these participants. Historically, when calculating CLBR, it has been suggested to perform calculations under conservative and optimistic assumptions and to assume that the truth lies somewhere in between (Malizia et al. 2009, Stewart et al. 2011). The conservative scenario amounts to assuming that the people with missing outcome data did not have a baby. The optimistic scenario (which has been erroneously referred to as the 'optimal' scenario in some papers (De Neubourg et al. 2021)) amounts to assuming that people with missing outcome data are just as likely to have a baby as those with observed outcome data. This approach, of providing conservative and optimistic estimates of CLBR, is still used (De Neubourg et al. 2021). A variation on this strategy is to make different assumptions based on what is known about the reason for the missing outcome data. For example, we might assume that people who have missing outcome data because they ceased treatment of their own accord have similar outcomes to people for whom outcomes are observed while assuming that people who were advised to stop treatment due to poor prognosis do not have a baby (Verhagen et al. 2008). More recently, an inverse probability weighting approach has been described which attempts to adjust the estimate of CLBR according to the measured prognostic characteristics of patients with missing outcome data (Modest et al. 2018). Whether using inverse probability weighting or an alternative method (such as regression), adjustment for prognostic variables can improve the credibility of missing data assumptions (see White et al. 2011, 2012) for practical advice and further discussion of this point).

An important note is that much of the methodological discussion concerning handling of missing outcomes in CLBR has considered the case where we wish to estimate the CLBR in a single cohort; the preceding paragraphs are no exception. In this situation, the designations 'conservative' and 'optimistic' are appropriate. However, extra care needs to be taken when we wish to compare outcomes between study arms (as we do in any reasonable study of the effect of an intervention). Suppose, in a two-arm study, that many more people drop out and have missing outcome data in the control arm than in the treatment arm. Assuming that anyone with missing data did not have a baby would give conservative estimates of the CLBRs in the two arms but would not provide a conservative estimate of the relative effectiveness of the experimental treatment compared to the control. It is advised to carry out a primary analysis based on one set of plausible assumptions about missing outcome data and to conduct a range of sensitivity analyses to assess the robustness of the result to alternative assumptions (White et al. 2011).

A subtle point to consider when choosing which assumptions to make about participants with missing outcome data is whether we should be making an assumption about what their unobserved outcome, as a matter of fact, is (e.g. given that they have ceased treatment but may continue to have unprotected intercourse) or rather, what the outcome would have been (e.g. were they, contrary to fact, to have continued treatment) (Diggle et al. 2007). This depends on the specific question we are trying to answer. If we are interested in the effect of a treatment in practice (accepting, e.g. that people may prematurely cease a course of treatment for a variety of reasons), then we should make assumptions about the actual unobserved outcome in these participants. If instead, we are interested in the effect of treatment if adhered to (undergoing the full course of treatment as intended) then we must make assumptions about what the outcome would have been had they completed the treatment (Mallinckrodt et al. 2017). In the latter case, it would be necessary to treat the outcomes of participants for whom outcomes were observed but who did not complete treatment as though their outcomes were missing for the purpose of analysis.

While cumulative outcomes are frequently relevant in the study of fertility interventions, recent reviews suggest that relatively few RCTs report these (Wilkinson et al. 2016, Kemper et al. 2020). Funding restrictions might be cited here, although there are counterexamples where follow-up over 12 months has been funded (Prentice et al. 2020). One final unresolved challenge relates to the current variation in defining and calculating CLBR (Maheshwari et al. 2015).

\section{Errors in the analysis of time to live birth}

In recognition of the fact that fertility treatment is often emotionally and financially burdensome, there is a current interest in identifying treatments and treatment strategies

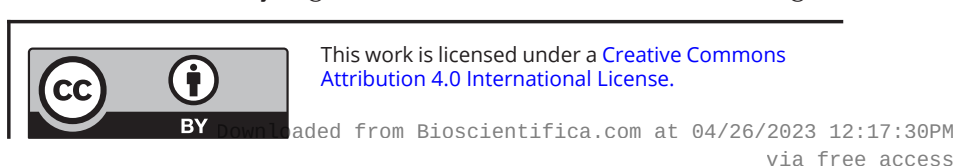


that reduce the duration from treatment initiation to live birth (Sunkara et al. 2020). This focus on a patientcentred outcome is laudable. We would urge some caution, however. Although interest in time to live birth in subfertility is relatively new, methodology for the analysis of time-to-event data is not and is both well established and routinely used in other clinical fields, such as oncology. This methodology must be applied in the context of infertility with due regard to clinical expertise, in order to ensure that any analytic assumptions are reasonable, but we would stress that there is no need to reinvent the wheel. It is concerning to see analyses of time to live birth, and indeed commentaries asserting how it should be done, which appear to be unaware of the appropriate techniques and which consequently fall into some common, but fatal traps.

One very natural but nonetheless completely erroneous approach, which has been used to suggest that PGT-A improves time to live birth, calculates the mean (or median) times using only the people who have had live births. The problems with this approach might not be immediately obvious, and indeed, this simple calculation might seem to be what is intended by the phrase 'median time to event'. However, it isn't. Figure 2 shows how this method can turn results on their head, suggesting an advantage of an inferior treatment. The figure shows participants in two treatment arms, A and B, undertaking up to three attempts at conception. The people coloured green have live births, and these are the only people included in the erroneous calculation. The people coloured yellow do not have live births after three attempts and are excluded from the erroneous calculation. Clearly, a

Restricting to births, we conclude mean/ median time to event is shorter in group A (absurd).

A

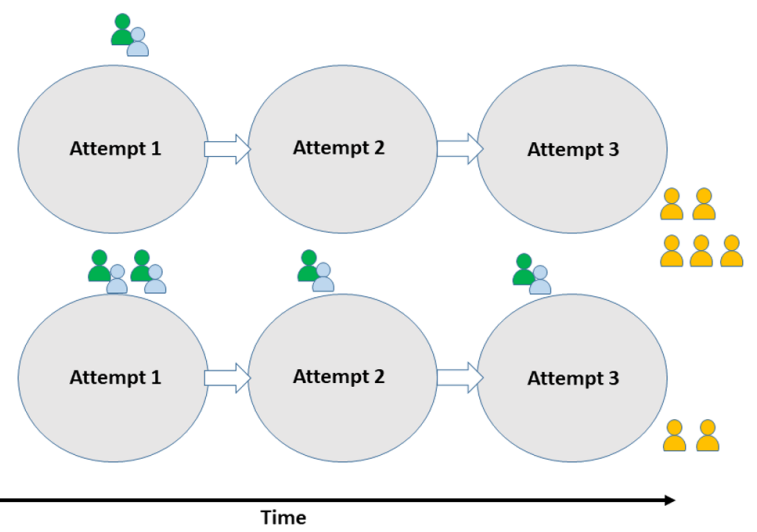

Figure 2 An illustration of why calculating 'time to live birth' using only people who had live births does not provide a valid estimate of a treatment's effect. patient can expect to have a live birth more quickly under treatment B than under treatment A. Under treatment A, most people (5/6) do not have a baby after three attempts, whereas under treatment B, most people (4/6) do. Yet the erroneous calculation will suggest that time to live birth is shorter under treatment $\mathrm{A}$, even though more people have live births in the first attempt under B. One final absurd consequence of the erroneous calculation is that 'time to live birth' will depend on follow-up time; the longer the duration of the study, the longer the calculated median (mean) time to event will be.

Appropriate analyses instead include the follow-up time of all participants, regardless of whether they had a live birth or not. The methods to be applied in this context are those developed for survival analysis, so-called because the event of interest in other clinical fields is often mortality. The methods can be applied to any scenario where we are interested in the timing of an event, however. An exposition of survival analysis is well beyond the scope of the present article, and we restrict ourselves to some considerations regarding the application to fertility data. We have previously provided a very brief introduction in the context of fertility research in the supplementary material (Duffy et al. 2020). We recommend a short series of tutorial papers from the British Journal of Cancer (Bradburn et al. 2003a,b, Clark et al. 2003a,b) for a more thorough introduction, and the textbook by Collett (Collett 2015) for a comprehensive treatment of the subject.

What is the median time to event, if not the median time taken to have the event amongst those who have it? It is instead the time taken for $50 \%$ of the cohort to have the event of interest. In many fertility studies, we do not anticipate that as many as half of the participants will have a baby within the follow-up period. It might, therefore, be more appropriate to report the time taken for some smaller proportion of participants (e.g. 25\%) to have live births. What about mean time to live birth? Although something like this could be calculated using appropriate statistical methods (Royston \& Parmar 2011, 2013), these methods are yet to be widely adopted, and many research studies are conducted without sufficient statistical expertise to use them. Consequently, we believe that use of the phrase 'mean time to live birth' often serves as a red flag; it frequently indicates that the erroneous calculation, described above, has been performed. We also note that, although it is common to speak of 'time to live birth', as we have done in this article, this is probably not the most clinically appropriate metric. This is because the time to live birth could be shortened by increasing the number of preterm births, which is not a desirable characteristic Attribution 4.0 International License. 
of a treatment. Several solutions are possible. One option, which is included in a core outcome set for infertility, is to use 'time to viable pregnancy leading to live birth', where the event is defined as detection of pregnancy with one or more heartbeats on ultrasound, which subsequently results in a live birth (Duffy et al. 2020). Other possibilities are to define the event as 'term live birth' or to use the due date rather than the actual date of birth.

A final consideration is how to measure 'time' in these analyses. Possibilities are to use continuous time (e.g. weeks, days) or to treat time as discrete units, such as 'number of attempts' (Daya 2005, Missmer et al. 2011). The appropriate choice is likely to depend on the particulars of the research question, although comparing treatments in terms of 'number of attempts' might obfuscate any differences in actual time (Daya 2005). For example, if we compare a freeze-all strategy in ART to a conventional strategy of fresh, followed by frozen transfers, then we need to be mindful that two transfer attempts can be made in the conventional arm in the time it takes to conduct one in the freeze-all arm (Zaat et al. 2019).

\section{Multiple treatment periods per participant}

In the preceding sections, we described the case where interventions influenced the outcome over multiple treatment attempts. In reality, we believe that this will usually be the case when assessing modern fertility treatments, particularly in the domain of ART. Our emphasis, therefore, differs slightly from previous authors who have described the case where an intervention is delivered repeatedly and may only affect the outcome of the next attempt. However, whenever this latter scenario does arise, the key statistical point is that the outcomes of repeated attempts undertaken by one individual (or couple) will tend to be more similar than those of several individuals (couples) (Vail \& Gardener 2003, Roberts \& Stylianou 2012, Missmer et al. 2011, Messerlian \& Gaskins 2017, Yland et al. 2019, Dodge et al. 2020). It is a mistake to analyse repeated treatment attempts undertaken by the same individual (couple) as though they represented attempts of different individuals (couples), as has been observed to happen in some RCTs (Vail \& Gardener 2003, Dias et al. 2008). In order to obtain valid statistical inferences, it is necessary to employ analytic methods capable of accommodating this correlation (Missmer et al. 2011, Roberts \& Stylianou 2012, Yland et al. 2019). A similar error occurs when participants contribute multiple oocytes or embryos to a dataset, and these are analysed ignoring any relatedness (Vail \& Gardener 2003). Further complexity arises due to the fact that the number of observations (attempts, oocytes, embryos) corresponding to each participant in the dataset is informative, requiring special treatment (Missmer et al. 2011, Yland et al. 2019). Performing appropriate analyses in this context requires a certain level of statistical competence, and since statistical experts are not the target of the current review, we do not go into any further detail here. Our recommendation to non-experts is to recognise the need for suitable expertise when conducting complex analyses; we direct statistical readers to the references appearing here and also to a review of methods for handling informative cluster size (Seaman et al. 2014).

Finally, we note that there is some controversy about the role of clinical trial designs which allow participants to be treated (and have an outcome assessment) on multiple occasions in the context of fertility research. These include crossover trials, in which participants are randomly allocated to receive each of the investigational and control interventions in a particular sequence (Senn 2002) and re-randomisation designs, in which participants are permitted to enter the study and be randomised to a treatment arm more than once (Kahan et al. 2015, 2018). If these designs are valid, they could reduce required sample sizes and improve recruitment to fertility RCTs, which would be a considerable benefit given the modest sizes of trials in the field (Stocking et al. 2019). The controversy arises because participants having a live birth typically do not proceed to receive further study treatment for subfertility, meaning, for example, that anyone having a live birth in the first period of a crossover trial would not proceed to the second period. It has been suggested both that valid inference is (Makubate \& Senn 2010, Budhram et al. 2019) and is not (Daya 1993, Vail \& Gardener 2003) possible under these circumstances. One practical consideration when applying these designs to fertility, which we have not seen discussed elsewhere, is that the outcome of previous treatment attempts will frequently be used to tailor the treatment delivered in a subsequent attempt. In ART, for example, the response to ovarian stimulation in a previous cycle is often used to modify the gonadotrophin dose and stimulation protocol in the next, and ICSI rather than IVF might be used if there were previous fertilisation issues. This might plausibly introduce a so-called carryover effect, which could be fatal to the validity of the crossover design, and consideration should be given to whether this sort of tailoring could be ethically prohibited. We speculate that tailoring on the basis of previous outcomes could also present a challenge for re-randomisation designs, if it influences the treatment

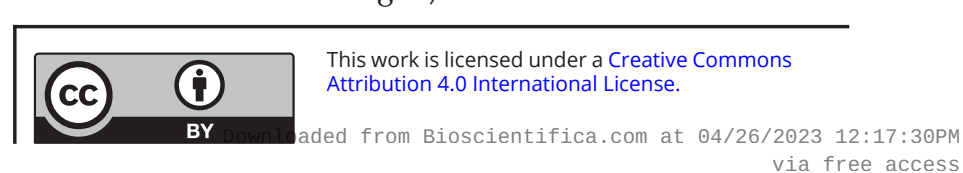


effect in such a way that it is no longer constant each time a participant is randomised (Kahan et al. 2015). Careful consideration of this point in relation to the study intervention would be necessary. At least one RCT employing re-randomisation in ART is underway at the time of writing (Bhide et al. 2020).

\section{Conclusions}

We have described some common methodological and statistical challenges arising in comparative effectiveness research in fertility. However, the issues we describe here are really just the tip of the iceberg. Various resources are available describing good statistical practice for biomedical research, but the quality is variable, with some making unfortunate errors. We recommend the Statistics notes series published by The BMJ (https://www.bmj.com/specialties/ statistics-notes ) and textbooks by Altman (Altman 1999) and Bland (Bland 2015) for accessible discussions of many topics. As we noted in the introduction, different statistical and design considerations apply to prognostic and diagnostic research. An excellent collection of resources relating to prognostic research, including the development of clinical prediction models, is available at https://www. prognosisresearch.com/.

Ultimately, we emphasise that medical statistics, which we take to include research design, is a deep, diverse and difficult subject, which can be studied for many years without achieving anything close to mastery. Whilewehope that this review will help non-statisticians to appreciate some recurring but consequential statistical errors, the best way to improve individual studies is to seek statistical input at an early stage. Noting the possible conflict of interest, we, therefore, advise researchers to include methodologists as collaborators where possible. This will frequently require that their contribution can be paid for since the salaries of many statisticians in academic departments are supported by grant income. Careful, thoughtful data analysis takes time, and while inclusion on a research output is nice (and where a substantial contribution has been made, warranted) the currency of co-authorship isn't acceptable as a salary for a key collaborator. Conversely, we stress that good medical research requires clinical as well as methodological expertise. We encourage applied statisticians to improve their own domain knowledge, as doing so facilitates discussion with clinical colleagues and helps to uncover methodological pitfalls which would otherwise be obfuscated by medical complexity. Indeed, statisticians who are not familiar with the field of fertility may not be alert to all of the methodological challenges we have described here. A meticulous dialogue between collaborators is, therefore, crucial for good study design; a last-second power calculation by email is not enough. Respectful collaboration between clinicians, methodologists, and partners with lived experience will remain the winning formula for useful fertility research.

\section{Declaration of interest}

JW declares that publishing in peer reviewed journals is likely to benefit his career.

\section{Funding}

JW is supported by a Wellcome Institutional Strategic Support Fund award [204796/Z/16/Z].

\section{Author contribution statement}

Both authors wrote the manuscript and approved the final version.

\section{Acknowledgements}

The authors thank to Daniel Brison for helpful comments on a draft of this manuscript. The idea to assess futility in a fertility trial using a surrogate endpoint such as pregnancy was originally suggested to us by Graeme MacLennan. Two anonymous peer reviewers also substantially improved the manuscript.

\section{References}

Abdalla HI, Bhattacharya S \& Khalaf Y 2010 Is meaningful reporting of national IVF outcome data possible? Human Reproduction 25 9-13. (https://doi.org/10.1093/humrep/dep357)

Al-Marzouki S, Roberts I, Evans S \& Marshall T 2008 Selective reporting in clinical trials: analysis of trial protocols accepted by the Lancet. Lancet 372 201. (https://doi.org/10.1016/S0140-6736(08)61060-0)

Althouse AD 2016 Adjust for multiple comparisons? It's not that simple. Annals of Thoracic Surgery 101 1644-1645 (https://doi.org/10.1016/j. athoracsur.2015.11.024).

Altman DG 1999 Practical Statistics for Medical Research. Boca Raton, Fla: Chapman \& Hall/CRC.

Bhide P, Srikantharajah A, Lanz D, Dodds J, Collins B, Zamora J, Chan D, Thangaratinam S \& Khan KS 2020 TILT: time-lapse imaging trial-a pragmatic, multi-centre, three-arm randomised controlled trial to assess the clinical effectiveness and safety of timelapse imaging in in vitro fertilisation treatment. Trials 21 600. (https:// doi.org/10.1186/s13063-020-04537-2)

Bland M 2015 An Introduction to Medical Statistics. Oxford: Oxford University Press.

Braakhekke M, Kamphuis EI, Dancet EA, Mol F, Van Der Veen F \& Mol BW 2014 Ongoing pregnancy qualifies best as the primary outcome measure of choice in trials in reproductive medicine: an opinion paper. Fertility and Sterility 101 1203-1204. (https://doi. org/10.1016/j.fertnstert.2014.03.047) 
Braakhekke M, Mol F, Mastenbroek S, Mol BW \& Van Der Veen F 2017a Equipoise and the RCT. Human Reproduction 32 257-260 (https://doi.org/10.1093/humrep/dew286).

Braakhekke M, Scholten I, Mol F, Limpens J, Mol BW \& Van Der Veen F 2017b Selective outcome reporting and sponsorship in randomized controlled trials in IVF and ICSI. Human Reproduction 32 2117-2122. (https://doi.org/10.1093/humrep/dex273)

Bradburn MJ, Clark TG, Love SB \& Altman DG 2003a Survival analysis part II: multivariate data analysis--an introduction to concepts and methods. British Journal of Cancer 89 431-436. (https:// doi.org/10.1038/sj.bjc.6601119)

Bradburn MJ, Clark TG, Love SB \& Altman DG 2003b Survival analysis part III: multivariate data analysis - choosing a model and assessing its adequacy and fit. British Journal of Cancer 89 605-611. (https://doi.org/10.1038/sj.bjc.6601120)

Bradburn MJ, Lee EC, White DA, Hind D, Waugh NR, Cooke DD, Hopkins D, Mansell P \& Heller SR 2020 Treatment effects may remain the same even when trial participants differed from the target population. Journal of Clinical Epidemiology 124 126-138. (https://doi. org/10.1016/j.jclinepi.2020.05.001)

Bratton DJ, Phillips PP \& Parmar MK 2013 A multi-arm multistage clinical trial design for binary outcomes with application to tuberculosis. BMC Medical Research Methodology 13 139. (https://doi. org/10.1186/1471-2288-13-139)

Budhram DR, Shi D, Mcdonald SD \& Walter SD 2019 The crossover design for studies of infertility employing in-vitro fertilization: a methodological survey. Contemporary Clinical Trials Communications 16 100426. (https://doi.org/10.1016/j.conctc.2019.100426)

Bujkiewicz S, Thompson JR, Riley RD \& Abrams KR 2016 Bayesian meta-analytical methods to incorporate multiple surrogate endpoints in drug development process. Statistics in Medicine 35 1063-1089. (https://doi.org/10.1002/sim.6776)

Chambers C 2019 What's next for registered reports? Nature 573 187-189. (https://doi.org/10.1038/d41586-019-02674-6)

Chambers CD, Dienes Z, Mcintosh RD, Rotshtein P \& Willmes K 2015 Registered reports: realigning incentives in scientific publishing. Cortex; A Journal Devoted to the Study of the Nervous System and Behavior 66 A1-A2. (https://doi.org/10.1016/j. cortex.2015.03.022)

Chiu YH, Stensrud MJ, Dahabreh IJ, Rinaudo P, Diamond MP, Hsu J, hernandez-diaz S \& Hernan MA 2020 The effect of prenatal treatments on offspring events in the presence of competing events: an application to a randomized trial of fertility therapies. Epidemiology 31 636-643. (https://doi.org/10.1097/EDE.0000000000001222)

Ciani O, Buyse M, Drummond M, Rasi G, Saad ED \& Taylor RS 2017 Time to review the role of surrogate end points in health policy: state of the art and the way forward. Value in Health 20 487-495. (https:// doi.org/10.1016/j.jval.2016.10.011)

Clark TG, Bradburn MJ, Love SB \& Altman DG 2003a Survival analysis part I: basic concepts and first analyses. British Journal of Cancer 89 232-238. (https://doi.org/10.1038/sj.bjc.6601118)

Clark TG, Bradburn MJ, Love SB \& Altman DG 2003b Survival analysis part IV: further concepts and methods in survival analysis. British Journal of Cancer 89 781-786. (https://doi.org/10.1038/ sj.bjc.6601117)

Collett D 2015 Modelling Survival Data in Medical Research. Boca Raton: CRC Press, Taylor \& Francis Group.

Craciunas L, Tsampras $\mathbf{N}$, Raine-Fenning $\mathbf{N} \&$ Coomarasamy $A$ 2018 Intrauterine administration of human chorionic gonadotropin (hCG) for subfertile women undergoing assisted reproduction. Cochrane Database of Systematic Reviews 10 CD011537. (https://doi. org/10.1002/14651858.CD011537)

Daya S 1993 Is there a place for the crossover design in infertility trials? Fertility and Sterility 59 6-7. (https://doi.org/10.1016/s00150282(16)55609-x)
Daya S 2005 Life table (survival) analysis to generate cumulative pregnancy rates in assisted reproduction: are we overestimating our success rates? Human Reproduction 20 1135-1143. (https://doi. org/10.1093/humrep/deh889)

De Neubourg D, Bogaerts K, Anagnostou E, Autin C, Blockeel C, Coetsier T, Delbaere A, Gillain N, Vandekerckhove F \& Wyns C 2021 Evolution of cumulative live birth and dropout rates over six complete IVF/ICSI cycles: a large prospective cohort study. Reproductive Biomedicine Online 42 717-724. (https://doi.org/10.1016/j. rbmo.2021.01.005)

Dias S, Mcnamee R \& Vail A 2008 Bias in frequently reported analyses of subfertility trials. Statistics in Medicine 27 5605-5619. (https://doi. org/10.1002/sim.3389)

Diggle P, Farewell D \& Henderson R 2007 Analysis of longitudinal data with drop-out: objectives, assumptions and a proposal. Journal of the Royal Statistical Society: Series C 56 499-550. (https://doi.org/10.1111/ j.1467-9876.2007.00590.x)

Dodge LE, Farland LV, Correia KFB, Missmer SA, Seidler EA, Wilkinson J, Modest AM \& Hacker MR 2020 Choice of statistical model in observational studies of ART. Human Reproduction 35 1499-1504. (https://doi.org/10.1093/humrep/deaa050)

Duffy JMN, Bhattacharya S, Bhattacharya S, Bofill M, Collura B, Curtis C, Evers JLH, Giudice LC, Farquharson RG, Franik S et al. 2020 Farquhar, C. M. \& core outcome measure for infertility trials, I. Human Reproduction 35 2735-2745. (https://doi. org/10.1093/humrep/deaa243)

Dwan K, Altman DG, Clarke M, Gamble C, Higgins JP, Sterne JA, Williamson PR \& Kirkham JJ 2014 Evidence for the selective reporting of analyses and discrepancies in clinical trials: a systematic review of cohort studies of clinical trials. PLoS Medicine 11 e1001666. (https://doi.org/10.1371/journal.pmed.1001666)

Easterbrook P 1987 Reducing publication bias. British Medical Journal 295 1347. (https://doi.org/10.1136/bmj.295.6609.1347-a)

Easterbrook PJ, Berlin JA, Gopalan R \& Matthews DR 1991 Publication bias in clinical research. Lancet 337 867-872. (https://doi. org/10.1016/0140-6736(91)90201-y)

European Medicines Agency 2016 Guideline on Multiplicity Issues in Clinical Trials. Committee for Human Medicinal Products.

Farland LV, Correia KF, Wise LA, Williams PL, Ginsburg ES \& Missmer SA 2016 P-values and reproductive health: what can clinical researchers learn from the American statistical association? Human Reproduction 31 2406-2410. (https://doi.org/10.1093/humrep/dew192)

Friede T, Stallard N \& Parsons N 2020 Adaptive seamless clinical trials using early outcomes for treatment or subgroup selection: methods, simulation model and their implementation in R. Biometrical Journal: Biometrische Zeitschrift 62 1264-1283. (https://doi.org/10.1002/ bimj.201900020)

Furukawa TA, Watanabe N, Omori IM, Montori VM \& Guyatt GH 2007 Association between unreported outcomes and effect size estimates in cochrane meta-analyses. Journal of the American Medical Association 297 468-470. (https://doi.org/10.1001/jama.297.5.468-b)

Goodman LK, Prentice LR, Chanati R \& Farquhar C 2020 Reporting assisted reproductive technology success rates on Australian and New Zealand fertility clinic websites. Australian and New Zealand Journal of Obstetrics and Gynaecology 60 135-140. (https://doi.org/10.1111/ ajo.13126)

Harbin Consensus Conference Workshop Group 2014 Improving the reporting of clinical trials of infertility treatments (IMPRINT): modifying the CONSORT statement. Fertility and Sterility 102 952-959. e15. (https://doi.org/10.1016/j.fertnstert.2014.08.002)

Heijnen EM, Macklon NS \& Fauser BC 2004 What is the most relevant standard of success in assisted reproduction? The next step to improving outcomes of IVF: consider the whole treatment. Human Reproduction 19 1936-1938. (https://doi.org/10.1093/humrep/ deh368) 
Hirji KF \& Fagerland MW 2009 Outcome based subgroup analysis: a neglected concern. Trials 1033 . (https://doi.org/10.1186/1745-621510-33)

Homer HA 2019 Preimplantation genetic testing for aneuploidy (PGT-A): the biology, the technology and the clinical outcomes. Australian and New Zealand Journal of Obstetrics and Gynaecology 59 317-324. (https:// doi.org/10.1111/ajo.12960)

Kahan BC, Forbes AB, Dore CJ \& Morris TP 2015 A re-randomisation design for clinical trials. BMC Medical Research Methodology 1596. (https://doi.org/10.1186/s12874-015-0082-2)

Kahan BC, Morris TP, Harris E, Pearse R, Hooper $R$ \& Eldridge $S$ 2018 Re-randomization increased recruitment and provided similar treatment estimates as parallel designs in trials of febrile neutropenia. Journal of Clinical Epidemiology 97 14-19. (https://doi.org/10.1016/j. jclinepi.2018.02.002)

Kemper JM, Wang R, Rolnik DL \& Mol BW 2020 Preimplantation genetic testing for aneuploidy: are we examining the correct outcomes? Human Reproduction 35 2408-2412. (https://doi. org/10.1093/humrep/deaa224)

Kleijkers SH, Mantikou E, Slappendel E, Consten D, Van Echten-Arends J, Wetzels AM, Van Wely M, Smits LJ, Van Montfoort AP, Repping S et al. 2016 Influence of embryo culture medium (G5 and HTF) on pregnancy and perinatal outcome after IVF: a multicenter RCT. Human Reproduction 31 2219-2230. (https://doi. org/10.1093/humrep/dew156)

Law YJ, Zhang N, Venetis CA, Chambers GM \& Harris K 2019 The number of oocytes associated with maximum cumulative live birth rates per aspiration depends on female age: a population study of 221 221 treatment cycles. Human Reproduction 34 1778-1787. (https://doi. org/10.1093/humrep/dez100)

Law YJ, Zhang N, Kolibianakis EM, Costello MF, Keller E, Chambers GM \& VENETIS CA 2021 Is there an optimal number of oocytes retrieved at which live birth rates or cumulative live birth rates per aspiration are maximized after ART? A systematic review. Reproductive Biomedicine Online 42 83-104. (https://doi.org/10.1016/j. rbmo.2020.10.008)

Lensen S, Chen S, Goodman L, Rombauts L, Farquhar C \& Hammarberg K 2021 IVF add-ons in australia and new zealand: a systematic assessment of IVF clinic websites. Australian and New Zealand Journal of Obstetrics and Gynaecology. (https://doi.org/10.1111/ajo.13321)

Lensen S, Martins W, Nastri C, Sadler L \& Farquhar C 2016 Pipelle for Pregnancy (PIP): study protocols for three randomised controlled trials. Trials 17 216. (https://doi.org/10.1186/s13063-016-1301-9)

Maheshwari A, Mclernon D \& Bhattacharya S 2015 Cumulative live birth rate: time for a consensus? Human Reproduction 30 2703-2707. (https://doi.org/10.1093/humrep/dev263)

Makubate B \& Senn S 2010 Planning and analysis of cross-over trials in infertility. Statistics in Medicine 29 3203-3210. (https://doi. $\operatorname{org} / 10.1002 / \operatorname{sim} .3981)$

Malizia BA, Hacker MR \& Penzias AS 2009 Cumulative live-birth rates after in vitro fertilization. New England Journal of Medicine $\mathbf{3 6 0}$ 236-243. (https://doi.org/10.1056/NEJMoa0803072)

Mallinckrodt C, Molenberghs G \& Rathmann S 2017 Choosing estimands in clinical trials with missing data. Pharmaceutical Statistics 16 29-36. (https://doi.org/10.1002/pst.1765)

Messerlian C \& Gaskins AJ 2017 Epidemiologic approaches for studying assisted reproductive technologies: design, methods, analysis and interpretation. Current Epidemiology Reports 4 124-132. (https://doi. org/10.1007/s40471-017-0105-0)

Miller D, Pavitt S, Sharma V, Forbes G, Hooper R, Bhattacharya S, Kirkman-Brown J, Coomarasamy A, Lewis S, Cutting $R$ et al. 2019 Physiological, hyaluronan-selected intracytoplasmic sperm injection for infertility treatment (HABSelect): a parallel, two-group, randomised trial. Lancet 393 416-422. (https://doi.org/10.1016/S01406736(18)32989-1)
Min JK, Breheny SA, Maclachlan V \& Healy DL 2004 What is the most relevant standard of success in assisted reproduction? The singleton, term gestation, live birth rate per cycle initiated: the BESST endpoint for assisted reproduction. Human Reproduction 19 3-7. (https://doi.org/10.1093/humrep/deh028)

Missmer SA, Pearson KR, Ryan LM, Meeker JD, Cramer DW \& Hauser R 2011 Analysis of multiple-cycle data From couples undergoing in vitro fertilization methodologic issues and statistical approaches. Epidemiology 22 497-504. (https://doi.org/10.1097/ EDE.0b013e31821b5351)

Modest AM, Wise LA, Fox MP, Weuve J, Penzias AS \& Hacker MR 2018 IVF success corrected for drop-out: use of inverse probability weighting. Human Reproduction 33 2295-2301. (https://doi. org/10.1093/humrep/dey309)

Moher D, Hopewell S, Schulz KF, Montori V, Gotzsche PC, Devereaux PJ, Elbourne D, Egger M \& Altman DG 2010 CONSORT 2010 Explanation and Elaboration: updated guidelines for reporting parallel group randomised trials. BMJ 340 c869-c869. (https://doi.org/10.1136/bmj.c869)

Munne S, Kaplan B, Frattarelli JL, Child T, Nakhuda G, Shamma FN, Silverberg K, Kalista T, Handyside AH, KatzJaffe M et al. 2019 Preimplantation genetic testing for aneuploidy versus morphology as selection criteria for single frozen-thawed embryo transfer in good-prognosis patients: a multicenter randomized clinical trial. Fertility and Sterility 112 1071.e7-1079.e7. (https://doi. org/10.1016/j.fertnstert.2019.07.1346)

Prentice L, Sadler L, Lensen S, Vercoe M, Wilkinson J, Edlin R, Chambers GM \& Farquhar CM 2020 IVF and IUI in couples with unexplained infertility (FIIX study): study protocol of a non-inferiority randomized controlled trial. Human Reproduction Open $\mathbf{2 0 2 0}$ hoaa037. (https://doi.org/10.1093/hropen/hoaa037)

Roberts SA \& Stylianou C 2012 The non-independence of treatment outcomes from repeat IVF cycles: estimates and consequences. Human Reproduction 27 436-443. (https://doi.org/10.1093/humrep/der420)

Roberts SA \& Vail A 2017 On the appropriate interpretation of evidence: the example of culture media and birth weight. Human Reproduction 32 1151-1154. (https://doi.org/10.1093/humrep/dex081)

Royston P \& Parmar MK 2011 The use of restricted mean survival time to estimate the treatment effect in randomized clinical trials when the proportional hazards assumption is in doubt. Statistics in Medicine 30 2409-2421. (https://doi.org/10.1002/sim.4274)

Royston P \& Parmar MK 2013 Restricted mean survival time: an alternative to the hazard ratio for the design and analysis of randomized trials with a time-to-event outcome. BMC Medical Research Methodology 13 152. (https://doi.org/10.1186/1471-2288-13-152)

Seaman S, Pavlou M \& Copas A 2014 Review of methods for handling confounding by cluster and informative cluster size in clustered data. Statistics in Medicine 33 5371-5387. (https://doi.org/10.1002/sim.6277)

Senn S 2002 Cross-Over Trials in Clinical Research. Chichester: Eng; New York: J. Wiley.

Simmons JP, Nelson LD \& Simonsohn U 2011 False-positive psychology: undisclosed flexibility in data collection and analysis allows presenting anything as significant. Psychological Science 22 1359-1366. (https://doi.org/10.1177/0956797611417632)

Snowden JM, Reavis KM \& Odden MC 2020 Conceiving of questions Before delivering analyses: relevant question formulation in reproductive and perinatal epidemiology. Epidemiology 31 644-648. (https://doi.org/10.1097/EDE.0000000000001223)

Stewart LM, Holman CD, Hart R, Finn J, Mai Q \& Preen DB 2011 How effective is in vitro fertilization, and how can it be improved? Fertility and Sterility 95 1677-1683. (https://doi.org/10.1016/j. fertnstert.2011.01.130).

Stocking K, Wilkinson J, Lensen S, Brison DR, Roberts SA \& Vail A 2019 Are interventions in reproductive medicine assessed for plausible and clinically relevant effects? A systematic review of power and

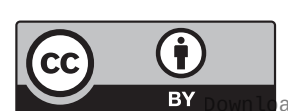

This work is licensed under a Creative Commons Attribution 4.0 International License. 
precision in trials and meta-analyses. Human Reproduction 34 659-665. (https://doi.org/10.1093/humrep/dez017)

Sunkara SK, Rittenberg V, Raine-Fenning N, Bhattacharya S, Zamora J \& Coomarasamy A 2011 Association between the number of eggs and live birth in IVF treatment: an analysis of 400 135 treatment cycles. Human Reproduction 26 1768-1774. (https://doi. org/10.1093/humrep/der106)

Sunkara SK, Zheng W, D'hooghe T, Longobardi S \& Boivin J 2020 Time as an outcome measure in fertility-related clinical studies: longawaited. Human Reproduction 35 1732-1739. (https://doi.org/10.1093/ humrep/deaa138)

Svensson S, Menkes DB \& Lexchin J 2013 Surrogate outcomes in clinical trials: a cautionary tale. Journal of the American Medical Association Internal Medicine 173 611-612. (https://doi.org/10.1001/ jamainternmed.2013.3037)

Vail A \& Gardener E 2003 Common statistical errors in the design and analysis of subfertility trials. Human Reproduction 18 1000-1004. (https://doi.org/10.1093/humrep/deg133)

Van De Wiel L, Wilkinson J, Athanasiou P \& Harper J 2020 The prevalence, promotion and pricing of three IVF add-ons on fertility clinic websites. Reproductive Biomedicine Online 41 801-806. (https:// doi.org/10.1016/j.rbmo.2020.07.021)

Van Rumste MM, Evers JL \& Farquhar CM 2003 Intra-cytoplasmic sperm injection versus conventional techniques for oocyte insemination during in vitro fertilisation in patients with non-male subfertility. Cochrane Database of Systematic Reviews (2) CD001301. (https://doi.org/10.1002/14651858.CD001301)

Verhagen TE, Dumoulin JC, Evers JL \& Land JA 2008 What is the most accurate estimate of pregnancy rates in IVF dropouts? Human Reproduction 23 1793-1799. (https://doi.org/10.1093/humrep/den209)

Wang R, Chen ZJ, Vuong LN, Legro RS, Mol BW \& Wilkinson J 2020 Large randomized controlled trials in infertility. Fertility and Sterility 113 1093-1099. (https://doi.org/10.1016/j.fertnstert.2020.04.037)

White IR, Horton NJ, Carpenter J \& Pocock SJ 2011 Strategy for intention to treat analysis in randomised trials with missing outcome data. BMJ 342 d40. (https://doi.org/10.1136/bmj.d40)

White IR, Carpenter J \& Horton NJ 2012 Including all individuals is not enough: lessons for intention-to-treat analysis. Clinical Trials $\mathbf{9}$ 396-407. (https://doi.org/10.1177/1740774512450098)

Wilkinson J, Roberts SA, Showell M, Brison DR \& Vail A 2016 No common denominator: a review of outcome measures in IVF RCTs.
Human Reproduction 31 2714-2722. (https://doi.org/10.1093/humrep/ dew227)

Wilkinson J, Roberts SA \& Vail A 2017 Developments in IVF warrant the adoption of new performance indicators for ART clinics, but do not justify the abandonment of patient-centred measures. Human Reproduction 32 1155-1159. (https://doi.org/10.1093/humrep/ dex063)

Wilkinson J, Brison DR, Duffy JMN, Farquhar CM, Lensen S, Mastenbroek S, Van Wely M \& Vail A 2019a Don't abandon RCTs in IVF. We don't even understand them. Human Reproduction 34 2093-2098. (https://doi.org/10.1093/humrep/dez199)

Wilkinson J, Pellicer A \& Niederberger C 2019b Registered reports: prospective peer review emphasizes science over spin. Fertility and Sterility 111 831-832. (https://doi.org/10.1016/j. fertnstert.2019.03.010)

Wilkinson J, Huang J, Marsden A, Harhay M, Vail A \& Roberts SA 2020 The implications of outcome truncation in reproductive medicine RCTs: a simulation platform for trialists and simulation study. (Available at: arXiv:2010.05684)

Yland J, Messerlian C, Minguez-Alarcon L, Ford JB, Hauser R, Williams PL \& EARTH Study Team 2019 Methodological approaches to analyzing IVF data with multiple cycles. Human Reproduction 34 549-557. (https://doi.org/10.1093/humrep/dey374)

Yusuf S, Collins R \& Peto R 1984 Why do we need some large, simple randomized trials? Statistics in Medicine 3 409-422. (https://doi. org/10.1002/sim.4780030421)

Yusuf S, Wittes J, Probstfield J \& Tyroler HA 1991 Analysis and interpretation of treatment effects in subgroups of patients in randomized clinical-trials. Journal of the American Medical Association 266 93-98. (https://doi.org/10.1001/jama.266.1.93)

Zaat T, Mol F, Van Wely M, Wilkinson J \& Mastenbroek S 2019 Fresh versus frozen blastocyst transfer. Lancet 394 1227. (https://doi. org/10.1016/S0140-6736(19)31392-3)

Received in final form 28 April 2021

Accepted 21 May 2021

Accepted Manuscript published online 21 May 2021 https://raf.bioscientifica.com

https://doi.org/10.1530/RAF-21-0015 (c) 2021 The authors Published by Bioscientifica Ltd

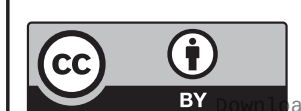

This work is licensed under a Creative Commons Attribution 4.0 International License. 